\title{
Can Estimation of Presepsin Levels in Endotracheal Aspirate Predict Early Onset Pneumonia in Newborns?
}

\author{
Shally Awasthi ${ }^{1}$ (1D \\ Received: 31 July 2018 / Accepted: 31 July 2018 /Published online: 11 August 2018 \\ (C) Dr. K C Chaudhuri Foundation 2018
}

Pneumonia is a major cause of mortality in children less than five years of age, the greatest risk being in the neonatal period. In neonates pneumonia can be of early onset, that is occurring within 7 days of birth or some have also suggested within first $48 \mathrm{~h}$, or late onset. Early onset pneumonia was found at autopsy in 10 $38 \%$ of stillborn and $20-63 \%$ of liveborns who subsequently died [1]. Mortality has been associated with birth weight and age of onset, with much higher case fatality rates in early than late onset pneumonia. Clinical predictive factors in mother or newborn may be absent in many cases of early onset pneumonia. However, there is no consensus definition of early onset pneumonia but a working definition has been proposed but not yet validated [2]. Sepsis screen had high co-positivity with positive blood culture and chest X-ray consistent with early onset pneumonia.

Presepsin is a free fragment of glycoprotein expressed on monocytes and macrophages and has been recognised as a biomarker of sepsis is neonates as well as in adults [3, 4]. A recent metaanalysis reported that serum presepsin had greater sensitivity than C-reactive protein and procalcitonin for detection of sepsis [5]. In this issue of the journal, Savić et al. [6] studied 60 neonates $\geq 24$ wk of gestation who were intubated and mechanically ventilated on the first day of life. These neonates were then classified into those with suspected pneumonia $(n=36)$ and others without pneumonia $(n=26)$, who were also control group. Presepsin levels were estimated in blood and tracheal aspirate samples and these were found to be statistically significantly higher in both fluids in cases of pneumonia. The authors attributed early onset pneumonia to acute intrauterine infection in their discussion.

In the publication by Savić et al. [6], the working definition of pneumonia was any two positive out of three criteria, clinical, radiological and microbiological, that is blood culture. However the distribution of these among cases and controls has not been

Shally Awasthi

shally07@gmail.com

1 Department of Pediatrics, King George's Medical University, Lucknow, India given. Also quality control of assessment and classification has not been mentioned. Since early onset pneumonia in neonates is usually accompanied by sepsis, it would have been appropriate to apply sepsis screen [2] on all subjects and report the copositivity rates. Cases were hospitalized neonates on mechanical ventilation and hence are different from the usual cases who primarily do not require ventilation in the early course of the disease. Hence, this effects the generalizability of results to other settings. There could also have been a selection bias while recruiting controls since authors did it by convenience sampling.

This study does demonstrate that tracheal aspirate presepsis levels may be used to identify early onset pneumonia. However, its performance against conventionally acceptable tools, which are economical and readily available, is lacking. Also this study has been done on sick and hospitalized neonates who were already getting optimal care. Hence further validation studies would be needed to establish diagnostic accuracy of tracheal presepsin levels in diagnosing early neonatal pneumonia.

\section{Compliance with Ethical Standards}

Conflict of Interest None.

\section{References}

1. Duke T. Neonatal pneumonia in developing countries. Arch Dis Child Fetal Neonatal Ed. 2005;90:F211-9.

2. Mathur NB, Garg K, Kumar S. Respiratory distress in neonates with special reference to pneumonia. Indian Pediatr. 2002;39:529-37.

3. Tabl HA, Abed NT. Diagnostic value of presepsin in neonatal sepsis. Egypt J Immunol. 2016;23:29-37.

4. Wu CC, Lan HM, Han ST, et al. Comparison of diagnostic accuracy in sepsis between presepsin, procalcitonin, and C-reactive protein: a systematic review and meta-analysis. Ann Intensive Care. 2017;7:91.

5. Bellos I, Fitrou G, Pergialiotis V, Thomakos N, Perrea DN, Daskalakis G. The diagnostic accuracy of presepsin in neonatal sepsis: a metaanalysis. Eur J Pediatr. 2018;177:625-32.

6. Savić D, Simović A, Marković S, et al. The role of presepsin obtained from tracheal aspirates in the diagnosis of early onset pneumonia in intubated newborns. Indian J Pediatr. 2018; https://doi.org/10. 1007/s12098-018-2676-2. 